\title{
IMPROVING THE AVERAGING THEORY FOR COMPUTING PERIODIC SOLUTIONS OF THE DIFFERENTIAL EQUATIONS
}

\author{
JAUME LLIBRE ${ }^{1}$ AND DOUGLAS D. NOVAES ${ }^{1,2}$
}

Abstract. For $m=1,2,3$, we consider differential systems of the form

$$
x^{\prime}=F_{0}(t, x)+\sum_{i=1}^{m} \varepsilon^{i} F_{i}(t, x)+\varepsilon^{m+1} R(t, x, \varepsilon),
$$

where $F_{i}: \mathbb{R} \times \mathcal{D} \rightarrow \mathbb{R}^{n}$, and $R: \mathbb{R} \times \mathcal{D} \times\left(-\varepsilon_{0}, \varepsilon_{0}\right) \rightarrow \mathbb{R}^{n}$ are $\mathcal{C}^{m+1}$ functions, and $T$-periodic in the first variable, being $\mathcal{D}$ an open subset of $\mathbb{R}^{n}$, and $\varepsilon$ a small parameter. For such system we assume that the unperturbed system $x^{\prime}=F_{0}(t, x)$ has a $k$-dimensional manifold of periodic solutions with $k \leq n$. We weaken the sufficient assumptions for studying the periodic solutions of the perturbed system when $|\varepsilon|>0$ is sufficiently small.

\section{IntRoduction AND STATEMENT OF the MAin RESUlts}

The averaging theory for computing periodic solutions of a differential system is one of the best analytical tools for the study of the periodic solutions, see for instance the papers $[23,13,19,12,14,17,18,20,10,11,8]$.

In the analysis of periodic solutions using the averaging theory there are essentially three main theorems. One for studying the periodic solutions of the periodic differential systems of the form $x^{\prime}=\varepsilon F(t, x, \varepsilon)$, with $x \in \mathbb{R}^{n}$ (see for instance $[26,24,2,9,15,16])$, and the other two for studying the periodic solutions of the periodic differential systems of the form $x^{\prime}=F_{0}(t, x)+\varepsilon F(t, x, \varepsilon)$ with $x \in \mathbb{R}^{n}$, distinguishing when the manifold $\mathcal{Z}$ of all periodic solutions of the unperturbed system $x^{\prime}=F_{0}(t, x)$ has dimension $n$ or smaller then $n$, respectively (see for instance $[21,22,3,4,9,15,16])$. The objective of this paper is to weaken the sufficient assumptions under the last system for studying their periodic solutions when the dimension of the manifold $\mathcal{Z}$ is smaller then $n$.

Let $\mathcal{D}$ be an open set of $\mathbb{R}^{n}$ and $\varepsilon_{0}>0$ be a small parameter. For $m=1,2,3$, we assume that the $\mathcal{C}^{m+1}$ functions $F_{i}: \mathbb{R} \times \mathcal{D} \rightarrow \mathbb{R}^{n}$ for $i=1, \ldots, m$ and $R: \mathbb{R} \times \mathcal{D} \times\left(-\varepsilon_{0}, \varepsilon_{0}\right) \rightarrow \mathbb{R}^{n}$ are $T$-periodic in the variable $t$. In what follows the prime denotes derivative with respect to the time $t$. Our study is concerned

2010 Mathematics Subject Classification. 37G15, 34C29, 37C30.

Key words and phrases. periodic solutions, limit cycles, averaging theory, Lyapunov-Schmidt reduction, nonlinear differential systems. 
about the existence of isolated $T$-periodic solutions of the differential systems of the form

$$
x^{\prime}=F_{0}(t, x)+\sum_{i=1}^{m} \varepsilon^{i} F_{i}(t, x)+\varepsilon^{m+1} R(t, x, \varepsilon),
$$

when there exists a $k$-dimensional submanifold $\mathcal{Z}$ of $\mathcal{D}(k \leq n)$ such that all solutions of the unperturbed system

$$
x^{\prime}=F_{0}(t, x),
$$

starting in $\mathcal{Z}$ are $T$-periodic. Formally, let $\beta: \bar{V} \rightarrow \mathbb{R}^{n-k}$ be a $\mathcal{C}^{m+1}$ function, with $V$ an open and bounded subset of $\mathbb{R}^{k}$ and let $\mathcal{Z}=\left\{z_{\alpha}=(\alpha, \beta(\alpha)): \alpha \in \bar{V}\right\}$. We shall assume that

$(H) \mathcal{Z} \subset \mathcal{D}$ and for each $z_{\alpha} \in \mathcal{Z}$ the unique solution $x_{\alpha}(t)=x\left(t, z_{\alpha}, 0\right)$ of $(2)$ such that $x_{\alpha}(0)=z_{\alpha}$ is $T$-periodic.

Here as usual $x(\cdot, z, \varepsilon):\left[0, t_{(z, \varepsilon)}\right) \rightarrow \mathbb{R}^{n}$ denotes the solution of system (1) such that $x(0, z, \varepsilon)=z$.

Let $Y(t, z)$ be a fundamental matrix solution of the linear differential system

$$
y^{\prime}=\frac{\partial F_{0}}{\partial x}(t, x(t, z, 0)) y \text {. }
$$

For each $z_{\alpha} \in \mathcal{Z}$ we denote $Y_{\alpha}(t)=Y\left(t, z_{\alpha}\right)$. System (3) is the linearization of system (2) through the solution $x(t, z, 0)$. For a given fundamental matrix solution $Y(t, z)$ we denote by $\Gamma_{\alpha}$ the upper right corner $k \times(n-k)$ matrix of $Y_{\alpha}(0)^{-1}-Y_{\alpha}(T)^{-1}$, and by $\Delta_{\alpha}$ the lower right corner $(n-k) \times(n-k)$ matrix of $Y_{\alpha}(0)^{-1}-Y_{\alpha}(T)^{-1}$.

Malkin [21] and Rosseau [22] have studied this problem up to order 1 in $\varepsilon$. Buică, Françoise and Llibre gave in [3] a simpler proof of their results when $\Gamma_{\alpha}$ is a null matrix for every $\alpha \in \mathcal{Z}$. Buică, Giné and Llibre in [4] have studied this problem up to order 2 in $\varepsilon$ also assuming that $\Gamma_{\alpha}$ is a null matrix for every $\alpha \in \mathcal{Z}$, and in [5] the same authors provided a nondegeneracy condition assuring the existence of a fundamental matrix solution $Y(t, z)$ for which $\Gamma_{\alpha}$ is a null matrix for every $\alpha \in \mathcal{Z}$. They also gave the formulae of the averaged function up to order 2 in $\varepsilon$ for this case. In [7] Rhouma and Chicone studied the same problem when the unperturbed system is autonomous.

In this work we give the formulae of the averaged functions up to order 3 in $\varepsilon$ without assuming that $\Gamma_{\alpha}$ is a null matrix. This approach represents a new way to deal with this kind of problem avoiding a previous treatment of the system. Moreover, as far as we know, the explicit formulae for the third order averaged function for systems like (1) are given for the first time in this present paper.

Choosing a system of coordinates where the matrix $\frac{\partial F_{0}}{\partial x}\left(t, x_{\alpha}(t)\right)$ is in its Jacobian normal form for every $t$ we obtain a system for which $\Gamma_{\alpha}$ is a null matrix. 
Unfortunately it is not always possible, as we can see in (9). Nevertheless it does not mean that the matrix $\Gamma_{\alpha}$ is different from zero for every system of coordinates and for every election of fundamental matrix solution $Y(t, z)$.

Given $p$ and $q$ positive integers let $G=\left(G_{1}, G_{2}, \ldots, G_{q}\right): \mathbb{R}^{p} \rightarrow \mathbb{R}^{q}$ be a smooth function, and consider the vectors $u=\left(u_{1}, u_{2}, \cdots, u_{p}\right) \in \mathbb{R}^{p}$ and $v^{i}=$ $\left(v_{1}^{i}, v_{2}^{i}, \cdots, v_{p}^{i}\right) \in \mathbb{R}^{p}$ for $i=1,2,3$. Thus for each $t \in \mathbb{R}$ and $\bar{u} \in \mathbb{R}^{p}$ we define

$$
\frac{\partial^{2} G}{\partial u^{2}}(t, \bar{u}) v^{1} v^{2}=\left(\sum_{i, j=1}^{p} \frac{\partial^{2} G_{1}}{\partial u_{i} \partial u_{j}}(t, \bar{u}) v_{i}^{1} v_{j}^{2}, \cdots, \sum_{i, j=1}^{p} \frac{\partial^{2} G_{q}}{\partial u_{i} \partial u_{j}}(t, \bar{u}) v_{i}^{1} v_{j}^{2}\right),
$$

(4) $\frac{\partial^{3} G}{\partial u^{3}}(t, \bar{u}) v^{1} v^{2} v^{3}=\left(\sum_{i, j, k=1}^{p} \frac{\partial^{2} G_{1}}{\partial u_{i} \partial u_{j} \partial u_{k}}(t, \bar{u}) v_{i}^{1} v_{j}^{2} v_{k}^{3}, \cdots\right.$,

$$
\left.\sum_{i, j=1}^{p} \frac{\partial^{2} G_{q}}{\partial u_{i} \partial u_{j} \partial u_{k}}(t, \bar{u}) v_{i}^{1} v_{j}^{2} v_{k}^{3}\right)
$$

We note that for each $t \in \mathbb{R}$ and $\bar{u} \in \mathbb{R}^{p}, \frac{\partial^{2} G}{\partial u^{2}}(t, \bar{u}): \mathbb{R}^{p} \times \mathbb{R}^{p} \rightarrow \mathbb{R}^{q}$ is a 2-linear map and $\frac{\partial^{3} G}{\partial u^{3}}(t, \bar{u}): \mathbb{R}^{p} \times \mathbb{R}^{p} \times \mathbb{R}^{p} \rightarrow \mathbb{R}^{q}$ is a 3-linear map.

In what follows the functions $\pi: \mathbb{R}^{k} \times \mathbb{R}^{n-k} \rightarrow \mathbb{R}^{k}$ and $\pi^{\perp}: \mathbb{R}^{k} \times \mathbb{R}^{n-k} \rightarrow \mathbb{R}^{n-k}$ will denote the projections onto the first $k$ coordinates and onto the last $n-k$ coordinates, respectively. For a point $z \in \mathcal{D}$ we also consider $z=(a, b) \in$ $\mathbb{R}^{k} \times \mathbb{R}^{n-k}$.

We define the averaged functions $f_{1}, f_{2}, f_{3}: \bar{V} \rightarrow \mathbb{R}^{k}$ as

$$
\begin{aligned}
f_{1}(\alpha)= & \Gamma_{\alpha} \gamma_{1}(\alpha)+\pi g_{1}\left(z_{\alpha}\right) \\
f_{2}(\alpha)= & \frac{1}{2} \Gamma_{\alpha} \gamma_{2}(\alpha)+\frac{1}{2} \frac{\partial^{2} \pi g_{0}}{\partial b^{2}}\left(z_{\alpha}\right) \gamma_{1}(\alpha)^{2}+\frac{\partial \pi g_{1}}{\partial b}\left(z_{\alpha}\right) \gamma_{1}(\alpha)+\pi g_{2}\left(z_{\alpha}\right), \\
f_{3}(\alpha)= & \frac{1}{6} \Gamma_{\alpha}\left(z_{\alpha}\right) \gamma_{3}(\alpha)+\frac{1}{6} \frac{\partial^{3} \pi g_{0}}{\partial b^{3}}\left(z_{\alpha}\right) \gamma_{1}(\alpha)^{3}+\frac{1}{2} \frac{\partial^{2} \pi g_{0}}{\partial b^{2}}\left(z_{\alpha}\right) \gamma_{1}(\alpha) \gamma_{2}(\alpha) \\
& +\frac{1}{2} \frac{\partial^{2} \pi g_{1}}{\partial b^{2}}\left(z_{\alpha}\right) \gamma_{1}(\alpha)^{2}+\frac{1}{2} \frac{\partial \pi g_{1}}{\partial b}\left(z_{\alpha}\right) \gamma_{2}(\alpha)+\frac{\partial \pi g_{2}}{\partial b}\left(z_{\alpha}\right) \gamma_{1}(\alpha) \\
& +\pi g_{3}\left(z_{\alpha}\right)
\end{aligned}
$$


where

$$
\begin{aligned}
\gamma_{1}(\alpha)= & -\Delta_{\alpha}^{-1} \pi^{\perp} g_{1}\left(z_{\alpha}\right) \\
\gamma_{2}(\alpha)= & -\Delta_{\alpha}^{-1}\left(\frac{\partial^{2} \pi^{\perp} g_{0}}{\partial b^{2}}\left(z_{\alpha}\right) \gamma_{1}(\alpha)^{2}+2 \frac{\partial \pi^{\perp} g_{1}}{\partial b}\left(z_{\alpha}\right) \gamma_{1}(\alpha)+2 \pi^{\perp} g_{2}\left(z_{\alpha}\right)\right), \\
\gamma_{3}(\alpha)= & -\Delta_{\alpha}^{-1}\left(\frac{\partial^{3} \pi^{\perp} g_{0}}{\partial b^{3}}\left(z_{\alpha}\right) \gamma_{1}(\alpha)^{3}+3 \frac{\partial^{2} \pi^{\perp} g_{0}}{\partial b^{2}}\left(z_{\alpha}\right) \gamma_{1}(\alpha) \gamma_{2}(\alpha)\right. \\
& +3 \frac{\partial^{2} \pi^{\perp} g_{1}}{\partial b^{2}}\left(z_{\alpha}\right) \gamma_{1}(\alpha)^{2}+2 \frac{\partial \pi^{\perp} g_{1}}{\partial b}\left(z_{\alpha}\right) \gamma_{2}(\alpha)+6 \frac{\partial \pi^{\perp} g_{2}}{\partial b}\left(z_{\alpha}\right) \gamma_{1}(\alpha) \\
& \left.+6 \pi^{\perp} g_{3}\left(z_{\alpha}\right)\right)
\end{aligned}
$$

and

$$
g_{i}(z)=Y(T, z)^{-1} \frac{y_{i}(T, z)}{i !},
$$

for $i=0,1,2,3$, being

(8)

$$
\begin{aligned}
y_{0}(t, z)= & x(t, z, 0)-z \\
y_{1}(t, z)= & Y(t, z) \int_{0}^{t} Y(\tau, z)^{-1} F_{1}(\tau, x(\tau, z, 0)) d \tau \\
y_{2}(t, z)= & Y(t, z) \int_{0}^{t} Y(\tau, z)^{-1}\left[2 F_{2}(\tau, x(\tau, z, 0))+2 \frac{\partial F_{1}}{\partial x}(\tau, x(\tau, z, 0))\right. \\
& \left.\cdot y_{1}(\tau, z)+\frac{\partial^{2} F_{0}}{\partial x^{2}}(\tau, x(\tau, z, 0)) y_{1}(\tau, z)^{2}\right] d \tau,
\end{aligned}
$$

$$
\begin{aligned}
y_{3}(t, z)= & Y(t, z) \int_{0}^{t} Y(\tau, z)^{-1}\left[6 F_{3}(\tau, x(\tau, z, 0))\right. \\
& +6 \frac{\partial F_{2}}{\partial x}(\tau, x(\tau, z, 0)) y_{1}(\tau, z)+3 \frac{\partial^{2} F_{1}}{\partial x^{2}}(\tau, x(\tau, z, 0)) y_{1}(\tau, z)^{2} \\
& +3 \frac{\partial F_{1}}{\partial x}(\tau, x(\tau, z, 0)) y_{2}(\tau, z)+3 \frac{\partial^{2} F_{0}}{\partial x^{2}}(\tau, x(\tau, z, 0)) y_{1}(\tau, z) \\
& \left.\cdot y_{2}(\tau, z)+\frac{\partial^{3} F_{0}}{\partial x^{3}}(\tau, x(\tau, z, 0)) y_{1}(\tau, z)^{3}\right] d \tau .
\end{aligned}
$$


Following the notation (4) the expression $\frac{\partial^{2} \pi g_{0}}{\partial b^{2}}\left(z_{\alpha}\right) \gamma_{1}(\alpha)^{2}$ in (5), is obtained taking $p=n-k, q=k, u=b, \bar{u}=\beta(\alpha), G: b \mapsto \pi g_{0}(\alpha, b)$, and $v^{1}=v^{2}=\gamma_{1}(\alpha)$. The similar expressions (6) and (8) are obtained analogously.

Our main result is given in the following theorem.

Theorem 1. In addition to hypothesis $(H)$ we assume that for each $z_{\alpha} \in \mathcal{Z}$, there exists a fundamental matrix solution of (3) such that $\operatorname{det}\left(\Delta_{\alpha}\right) \neq 0$ for every $\alpha \in \bar{V}$. So the following statements hold.

(a) Take $m=1$. If there exists $\alpha^{*} \in V$ with $f_{1}\left(\alpha^{*}\right)=0$ and $\operatorname{det}\left(D f_{1}\left(\alpha^{*}\right)\right) \neq 0$, then there exists a T-periodic solution $\varphi(t, \varepsilon)$ of system (1) such that $\varphi(0, \varepsilon) \rightarrow z_{\alpha^{*}}$ when $\varepsilon \rightarrow 0$.

(b) Take $m=2$ and assume that $f_{1}(\alpha) \equiv 0$. If there exists $\alpha^{*} \in V$ with $f_{2}\left(\alpha^{*}\right)=0$ and $\operatorname{det}\left(D f_{2}\left(\alpha^{*}\right)\right) \neq 0$, then there exists a T-periodic solution $\varphi(t, \varepsilon)$ of system $(1)$ such that $\varphi(0, \varepsilon) \rightarrow z_{\alpha^{*}}$ when $\varepsilon \rightarrow 0$.

(c) Take $m=3$ and assume that $f_{1}(\alpha) \equiv f_{2}(\alpha) \equiv 0$. If there exists $\alpha^{*} \in V$ with $f_{3}\left(\alpha^{*}\right)=0$ and $\operatorname{det}\left(D f_{3}\left(\alpha^{*}\right)\right) \neq 0$, then there exists a $T$-periodic solution $\varphi(t, \varepsilon)$ of system $(1)$ such that $\varphi(0, \varepsilon) \rightarrow z_{\alpha^{*}}$ when $\varepsilon \rightarrow 0$.

Theorem extends the main results of $[4,5]$, and it is proved in section 2 .

As an application of Theorem 1 we consider the following quadratic polynomial non-autonomous system of differential equations:

$$
\begin{aligned}
& u^{\prime}=w+\varepsilon \mu v^{2} \sin t+\varepsilon^{2} \nu, \\
& v^{\prime}=w+\varepsilon \eta v^{2} \sin t+\varepsilon^{2} \nu, \\
& w^{\prime}=(1+\cos t) w+\varepsilon^{2} \bar{\nu}+\varepsilon^{3} \xi \sin t .
\end{aligned}
$$

Let

$$
A_{0}(t)=\int_{0}^{t} e^{s+\sin s} d s, \quad \text { and } \quad A_{1}(t)=\int_{0}^{t} e^{-s-\sin s} A_{0}(s) \sin s d s .
$$

We assume that

$$
\bar{\nu}=\frac{2 \pi\left(e^{2 \pi}-1\right) \nu}{\left(e^{2 \pi}-1\right) A_{1}(2 \pi)-A_{0}(2 \pi) A_{0}(-2 \pi)} \approx 0.775 \nu .
$$

We note that for $\varepsilon=0$ the linear part of system (9) is given by the matrix

$$
\left(\begin{array}{ccc}
0 & 0 & 1 \\
0 & 0 & 1 \\
0 & 0 & 1+\cos t
\end{array}\right) .
$$


For $t \neq \pi$ the Jacobian normal form of (11) is given by

$$
\left(\begin{array}{ccc}
0 & 0 & 0 \\
0 & 0 & 0 \\
0 & 0 & 1+\cos t
\end{array}\right) .
$$

Nevertheless the Jacobian normal form of (11) when $t=\pi$ is given by

$$
\left(\begin{array}{lll}
0 & 0 & 0 \\
0 & 0 & 1 \\
0 & 0 & 0
\end{array}\right) .
$$

Hence there is no continuous change of variables which transforms the matrix (11) into (12).

In the next proposition we give sufficient conditions for the existence of an isolated periodic solution of system (9) when $|\varepsilon|>0$ is sufficiently small.

Proposition 2. If $\mu \eta \nu \neq 0$, then for $|\varepsilon|>0$ sufficiently small there exist an isolate periodic solution $\varphi(t, \varepsilon)$ of system $(9)$ such that $\varphi(0, \varepsilon) \rightarrow\left(u^{*}, v^{*}, 0\right) \in \mathcal{D}$ where

$$
\left(u^{*}, v^{*}\right)=k\left(\frac{\xi}{\eta \nu}, \frac{\xi}{\mu \nu}\right) \quad \text { and } \quad k \approx 2.952
$$

\section{Proofs of Theorem 1 and Proposition 2}

We shall need the next lemma to prove our main result. Lemma 3 extends Theorem 4 of [4] which is a particular case of the Lyapunov-Schmidt reduction method for finite dimensional function (see for instance [6]).

Lemma 3. Assume that $k \leq n$ are positive integers. Let $\mathcal{D}$ and $V$ be open subsets of $\mathbb{R}^{n}$ and $\mathbb{R}^{k}$, respectively. For $m=1,2,3$, let $g_{i}(z)$ for $i=0,1, \ldots, m$ and $\beta: \bar{V} \rightarrow \mathbb{R}^{n-k}$ be $\mathcal{C}^{m+1}$ functions and take $g: \mathcal{D} \times\left(-\varepsilon_{0}, \varepsilon_{0}\right) \rightarrow \mathbb{R}^{n}$ such that

$$
g(z, \varepsilon)=g_{0}(z)+\sum_{i=1}^{m} \varepsilon^{i} g_{i}(z)+\mathcal{O}\left(\varepsilon^{m+1}\right),
$$

and $\mathcal{Z}=\left\{z_{\alpha}=(\alpha, \beta(\alpha)): \alpha \in \bar{V}\right\} \subset \mathcal{D}$. We denote by $\Gamma_{\alpha}$ the upper right corner $k \times(n-k)$ matrix of $D g_{0}\left(z_{\alpha}\right)$, and by $\Delta_{\alpha}$ the lower right corner $(n-k) \times(n-k)$ matrix of $D g_{0}\left(z_{\alpha}\right)$. Assume that for each $z_{\alpha} \in \mathcal{Z}$, $\operatorname{det}\left(\Delta_{\alpha}\right) \neq 0$ and $g_{0}\left(z_{\alpha}\right)=0$. We consider the functions $f_{1}, f_{2}, f_{3}: \bar{V} \rightarrow \mathbb{R}^{k}$ defined in (5). Then the following statements hold.

(a) Take $m=1$. If there exists $\alpha^{*} \in V$ with $f_{1}\left(\alpha^{*}\right)=0$ and $\operatorname{det}\left(D f_{1}\left(\alpha^{*}\right)\right) \neq$ 0 , then there exists $\alpha_{\varepsilon}$ such that $g\left(z_{\alpha_{\varepsilon}}, \varepsilon\right)=0$ and $z_{\alpha_{\varepsilon}} \rightarrow z_{\alpha^{*}}$ when $\varepsilon \rightarrow 0$. 
(b) Take $m=2$ and assume that $f_{1}(\alpha) \equiv 0$. If there exists $\alpha^{*} \in V$ with $f_{2}\left(\alpha^{*}\right)=0$ and $\operatorname{det}\left(D f_{2}\left(\alpha^{*}\right)\right) \neq 0$, then there exists $\alpha_{\varepsilon}$ such that $g\left(z_{\alpha_{\varepsilon}}, \varepsilon\right)=$ 0 and $z_{\alpha_{\varepsilon}} \rightarrow z_{\alpha^{*}}$ when $\varepsilon \rightarrow 0$.

(c) Take $m=3$ and assume that $f_{1}(\alpha) \equiv f_{2}(\alpha) \equiv 0$. If there exists $\alpha^{*} \in V$ with $f_{3}\left(\alpha^{*}\right)=0$ and $\operatorname{det}\left(D f_{3}\left(\alpha^{*}\right)\right) \neq 0$, then there exists $\alpha_{\varepsilon}$ such that $g\left(z_{\alpha_{\varepsilon}}, \varepsilon\right)=0$ and $z_{\alpha_{\varepsilon}} \rightarrow z_{\alpha^{*}}$ when $\varepsilon \rightarrow 0$.

Note that in the Lemma 3 the functions $g_{i}$ for $i=0,1,2,3$, which appear in the expression of (5) and (6) are the ones of the function (14), instead of the functions which appear in (7).

Proof of Lemma 3. First of all we consider $g=\left(\pi g, \pi^{\perp} g\right), g_{i}=\left(\pi g_{i}, \pi^{\perp} g_{i}\right)$ for $i=0,1,2$, and $z=(a, b) \in \mathbb{R}^{k} \times \mathbb{R}^{n-k}$ for $z \in \mathcal{D}$. So

$$
\frac{\partial g}{\partial z}\left(z_{\alpha}, 0\right)=D g_{0}\left(z_{\alpha}\right)=\left(\begin{array}{cc}
\frac{\partial \pi g_{0}}{\partial a}\left(z_{\alpha}\right) & \frac{\partial \pi g_{0}}{\partial b}\left(z_{\alpha}\right) \\
\frac{\partial \pi^{\perp} g_{0}}{\partial a}\left(z_{\alpha}\right) & \frac{\partial \pi^{\perp} g_{0}}{\partial b}\left(z_{\alpha}\right)
\end{array}\right)
$$

We note that $\Gamma_{\alpha}=\frac{\partial \pi g_{0}}{\partial b}\left(z_{\alpha}\right)$ and $\Delta_{\alpha}=\frac{\partial \pi^{\perp} g_{0}}{\partial b}\left(z_{\alpha}\right)$.

From the hypotheses we have $\pi^{\perp} g(\alpha, \beta(\alpha), 0)=\pi^{\perp} g_{0}\left(z_{\alpha}\right)=0$ and

$$
\operatorname{det}\left(\frac{\partial \pi^{\perp} g}{\partial b}(\alpha, \beta(\alpha), 0)\right)=\operatorname{det}\left(\frac{\partial \pi^{\perp} g_{0}}{\partial b}\left(z_{\alpha}\right)\right)=\operatorname{det}\left(\Delta_{\alpha}\right) \neq 0
$$

Thus applying the Implicit Function Theorem (see for instance [25]) it follows that there exists an open neighbourhood $U \times\left(-\varepsilon_{1}, \varepsilon_{1}\right)$ of $\bar{V} \times\{0\}$ with $\varepsilon_{1} \leq \varepsilon_{0}$, and a $\mathcal{C}^{m+1}$ function $\bar{\beta}: U \times\left(-\varepsilon_{1}, \varepsilon_{1}\right) \rightarrow \mathbb{R}^{n-k}$ such that $\pi^{\perp} g(a, \bar{\beta}(a, \varepsilon), \varepsilon)=0$ for each $(a, \varepsilon) \in U \times\left(-\varepsilon_{1}, \varepsilon_{1}\right)$ and $\bar{\beta}(\alpha, 0)=\beta(\alpha)$ for every $\alpha \in \bar{V}$. Here the value 
of $m$ depends on the statement $(a),(b)$ or $(c)$ we are proving. So we compute

$$
\begin{aligned}
\frac{\partial \bar{\beta}}{\partial \varepsilon}(\alpha, 0)= & -\Delta_{\alpha}^{-1} \pi^{\perp} g_{1}\left(z_{\alpha}\right)=\gamma_{1}(\alpha) \\
\frac{\partial^{2} \bar{\beta}}{\partial \varepsilon^{2}}(\alpha, 0)= & -\Delta_{\alpha}^{-1}\left(\frac{\partial^{2} \pi^{\perp} g_{0}}{\partial b^{2}}\left(z_{\alpha}\right) \gamma_{1}(\alpha)^{2}\right. \\
& \left.+2 \frac{\partial \pi^{\perp} g_{1}}{\partial b}\left(z_{\alpha}\right) \gamma_{1}(\alpha)+2 \pi^{\perp} g_{2}\left(z_{\alpha}\right)\right)=\gamma_{2}(\alpha) \\
\frac{\partial^{3} \bar{\beta}}{\partial \varepsilon^{2}}(\alpha, 0)= & -\Delta_{\alpha}^{-1}\left(\frac{\partial^{3} \pi^{\perp} g_{0}}{\partial b^{3}}\left(z_{\alpha}\right) \gamma_{1}(\alpha)^{3}+3 \frac{\partial^{2} \pi^{\perp} g_{0}}{\partial b^{2}}\left(z_{\alpha}\right) \gamma_{1}(\alpha) \gamma_{2}(\alpha)\right. \\
& +3 \frac{\partial^{2} \pi^{\perp} g_{1}}{\partial b^{2}}\left(z_{\alpha}\right) \gamma_{1}(\alpha)^{2}+2 \frac{\partial \pi^{\perp} g_{1}}{\partial b}\left(z_{\alpha}\right) \gamma_{2}(\alpha) \\
& \left.+6 \frac{\partial \pi^{\perp} g_{2}}{\partial b}\left(z_{\alpha}\right) \gamma_{1}(\alpha)+6 \pi^{\perp} g_{3}\left(z_{\alpha}\right)\right)=\gamma_{3}(\alpha) .
\end{aligned}
$$

Now for each $\alpha \in \bar{V}$ we consider the $\mathcal{C}^{m+1}$ function $\delta: \varepsilon \mapsto \pi g(\alpha, \bar{\beta}(\alpha, \varepsilon), \varepsilon) \in$ $\mathbb{R}^{k}$. We know that

$$
\delta(\alpha, \varepsilon)=\delta(\alpha, 0)+\varepsilon \frac{\partial \delta}{\partial \varepsilon}(\alpha, 0)+\varepsilon^{2} \frac{1}{2} \frac{\partial^{2} \delta}{\partial \varepsilon^{2}}(\alpha, 0)+\varepsilon^{3} \frac{1}{6} \frac{\partial^{3} \delta}{\partial \varepsilon^{3}}(\alpha, 0)+\mathcal{O}\left(\varepsilon^{4}\right)
$$

We observe that the above expression is written assuming that $m=3$. If $m=2$ it must be truncated in $\mathcal{O}\left(\varepsilon^{2}\right)$, and if $m=1$ it must be truncated in $\mathcal{O}\left(\varepsilon^{2}\right)$.

We compute

$$
\begin{aligned}
\frac{\partial \delta}{\partial \varepsilon}(\alpha, \varepsilon)= & \frac{\partial \pi g}{\partial b}(\alpha, \bar{\beta}(\alpha, \varepsilon), \varepsilon) \frac{\partial \bar{\beta}}{\partial \varepsilon}(\alpha, \varepsilon)+\frac{\partial \pi g}{\partial \varepsilon}(\alpha, \bar{\beta}(\alpha, \varepsilon), \varepsilon) \\
\frac{\partial^{2} \delta}{\partial \varepsilon^{2}}(\alpha, \varepsilon)= & \frac{\partial^{2} \pi g}{\partial b^{2}}(\alpha, \bar{\beta}(\alpha, \varepsilon), \varepsilon)\left(\frac{\partial \bar{\beta}}{\partial \varepsilon}(\alpha, \varepsilon)\right)^{2} \\
& +2 \frac{\partial^{2} \pi g}{\partial \varepsilon \partial b}(\alpha, \bar{\beta}(\alpha, \varepsilon), \varepsilon) \frac{\partial \bar{\beta}}{\partial \varepsilon}(\alpha, \varepsilon)+\frac{\partial \pi g}{\partial b}(\alpha, \bar{\beta}(\alpha, \varepsilon), \varepsilon) \frac{\partial^{2} \bar{\beta}}{\partial \varepsilon^{2}}(\alpha, \varepsilon) \\
& +\frac{\partial^{2} \pi g}{\partial \varepsilon^{2}}(\alpha, \bar{\beta}(\alpha, \varepsilon), \varepsilon)
\end{aligned}
$$




$$
\begin{aligned}
\frac{\partial^{3} \delta}{\partial^{3} \varepsilon}(\alpha, \varepsilon)= & \frac{\partial^{3} \pi g}{\partial b^{3}}(\alpha, \bar{\beta}(\alpha, \varepsilon), \varepsilon)\left(\frac{\partial \bar{\beta}}{\partial \varepsilon}(\alpha, \varepsilon)\right)^{3}+3 \frac{\partial^{2} \pi g}{\partial b^{2}}(\alpha, \bar{\beta}(\alpha, \varepsilon), \varepsilon) \frac{\partial \bar{\beta}}{\partial \varepsilon}(\alpha, \varepsilon) \\
& \cdot \frac{\partial^{2} \bar{\beta}}{\partial \varepsilon^{2}}(\alpha, \varepsilon)+\frac{\partial \pi g}{\partial b}(\alpha, \bar{\beta}(\alpha, \varepsilon), \varepsilon) \frac{\partial^{3} \bar{\beta}}{\partial \varepsilon^{3}}(\alpha, \varepsilon) \\
& +3 \frac{\partial^{3} \pi g}{\partial \varepsilon \partial b^{2}}(\alpha, \bar{\beta}(\alpha, \varepsilon), \varepsilon)\left(\frac{\partial \bar{\beta}}{\partial \varepsilon}(\alpha, \varepsilon)\right)^{2}+3 \frac{\partial^{2} \pi g}{\partial \varepsilon \partial b}(\alpha, \bar{\beta}(\alpha, \varepsilon), \varepsilon) \\
& \cdot \frac{\partial^{2} \bar{\beta}}{\partial \varepsilon^{2}}(\alpha, \varepsilon)+3 \frac{\partial^{3} \pi g}{\partial \varepsilon^{2} \partial b}(\alpha, \bar{\beta}(\alpha, \varepsilon), \varepsilon) \frac{\partial \bar{\beta}}{\partial \varepsilon}(\alpha, \varepsilon) \\
& +\frac{\partial^{3} \pi g}{\partial \varepsilon^{3}}(\alpha, \bar{\beta}(\alpha, \varepsilon), \varepsilon) .
\end{aligned}
$$

Thus

$$
\begin{aligned}
\delta(\alpha, 0)= & 0 \\
\frac{\partial \delta}{\partial \varepsilon}(\alpha, 0)= & \frac{\partial \pi g_{0}}{\partial b}\left(z_{\alpha}\right) \frac{\partial \bar{\beta}}{\partial \varepsilon}(\alpha, 0)+\pi g_{1}\left(z_{\alpha}\right) \\
= & \Gamma_{\alpha} \gamma_{1}(\alpha)+\pi g_{1}\left(z_{\alpha}\right)=f_{1}(\alpha) \\
\frac{\partial^{2} \delta}{\partial \varepsilon^{2}}(\alpha, 0)= & \frac{\partial^{2} \pi g_{0}}{\partial b^{2}}\left(z_{\alpha}\right)\left(\frac{\partial \bar{\beta}}{\partial \varepsilon}(\alpha, 0)\right)^{2}+2 \frac{\partial \pi g_{1}}{\partial b}\left(z_{\alpha}\right) \frac{\partial \bar{\beta}}{\partial \varepsilon}(\alpha, 0) \\
& +\frac{\partial \pi g_{0}}{\partial b}\left(z_{\alpha}\right) \frac{\partial^{2} \bar{\beta}}{\partial \varepsilon^{2}}(\alpha, 0)+2 \pi g_{2}\left(z_{\alpha}\right) \\
= & \Gamma_{\alpha} \gamma_{2}(\alpha)+\frac{\partial^{2} \pi g_{0}}{\partial b^{2}}\left(z_{\alpha}\right) \gamma_{1}(\alpha)^{2}+2 \frac{\partial \pi g_{1}}{\partial b}\left(z_{\alpha}\right) \gamma_{1}(\alpha) \\
& +2 \pi g_{2}\left(z_{\alpha}\right)=2 f_{2}(\alpha), \\
& +\frac{\partial^{3} \pi g_{0}}{\partial b^{3}}\left(z_{\alpha}\right)\left(\frac{\partial \bar{\beta}}{\partial \varepsilon}(\alpha, \varepsilon)\right)^{3}+3 \frac{\partial^{2} \pi g_{0}}{\partial b^{2}}\left(z_{\alpha}\right) \frac{\partial \bar{\beta}}{\partial \varepsilon}(\alpha, \varepsilon) \frac{\partial^{2} \bar{\beta}}{\partial \varepsilon^{2}}(\alpha, \varepsilon) \\
& \cdot \frac{\partial^{2} \bar{\beta}}{\partial \varepsilon^{2}}(\alpha, \varepsilon)+6 \frac{\partial \pi g_{2}}{\partial \varepsilon^{3}}(\alpha, \varepsilon)+3 \frac{\partial^{2} \pi g_{1}}{\partial b^{2}}\left(z_{\alpha}\right)\left(\frac{\partial \bar{\beta}}{\partial \varepsilon}(\alpha, \varepsilon)\right)^{2}+3 \frac{\partial \pi g_{1}}{\partial \varepsilon}(\alpha, \varepsilon)+6 \pi g_{3}\left(z_{\alpha}\right) \\
&
\end{aligned}
$$




$$
\begin{aligned}
= & \Gamma_{\alpha}\left(z_{\alpha}\right) \gamma_{3}(\alpha)+\frac{\partial^{3} \pi g_{0}}{\partial b^{3}}\left(z_{\alpha}\right) \gamma_{1}(\alpha)^{3}+3 \frac{\partial^{2} \pi g_{0}}{\partial b^{2}}\left(z_{\alpha}\right) \gamma_{1}(\alpha) \gamma_{2}(\alpha) \\
& +3 \frac{\partial^{2} \pi g_{1}}{\partial b^{2}}\left(z_{\alpha}\right) \gamma_{1}(\alpha)^{2}+3 \frac{\partial \pi g_{1}}{\partial b}\left(z_{\alpha}\right) \gamma_{2}(\alpha)+6 \frac{\partial \pi g_{2}}{\partial b}\left(z_{\alpha}\right) \gamma_{1}(\alpha) \\
& +6 \pi g_{3}\left(z_{\alpha}\right)=6 f_{3}(\alpha) .
\end{aligned}
$$

If $m=1$ and $f_{1}(\alpha) \not \equiv 0$, then the Implicit Function Theorem, applied to the function $\delta(\alpha, \varepsilon) / \varepsilon$ for $|\varepsilon|>0$ sufficiently small, guarantees the existence of $\alpha(\varepsilon)$ such that $\alpha(0)=\alpha^{*}$ and $\delta(\alpha(\varepsilon), \varepsilon)=0$. Denoting $z_{\alpha_{\varepsilon}}=(\alpha(\varepsilon), \bar{\beta}(\alpha(\varepsilon)), \varepsilon)$ we have $f\left(z_{\alpha_{\varepsilon}}, \varepsilon\right)=0$. So statement $(a)$ is proved.

If $m=2$ and $f_{1}(\alpha) \equiv 0$, then the Implicit Function Theorem, applied to the function $\delta(\alpha, \varepsilon) / \varepsilon^{2}$ for $|\varepsilon|>0$ sufficiently small, guarantees the existence of $\alpha(\varepsilon)$ such that $\alpha(0)=\alpha^{*}$ and $\delta(\alpha(\varepsilon), \varepsilon)=0$. Denoting $z_{\alpha_{\varepsilon}}=(\alpha(\varepsilon), \bar{\beta}(\alpha(\varepsilon)), \varepsilon)$ we have $f\left(z_{\alpha_{\varepsilon}}, \varepsilon\right)=0$. So statement $(b)$ is proved.

Finally If $m=3$ and $f_{1}(\alpha) \equiv f_{2}(\alpha) \equiv 0$, then the Implicit Function Theorem, applied to the function $\delta(\alpha, \varepsilon) / \varepsilon^{3}$ for $|\varepsilon|>0$ sufficiently small, guarantees the existence of $\alpha(\varepsilon)$ such that $\alpha(0)=\alpha^{*}$ and $\delta(\alpha(\varepsilon), \varepsilon)=0$. Denoting $z_{\alpha_{\varepsilon}}=$ $(\alpha(\varepsilon), \bar{\beta}(\alpha(\varepsilon)), \varepsilon)$ we have $f\left(z_{\alpha_{\varepsilon}}, \varepsilon\right)=0$. So statement $(c)$ is proved.

Proof of Theorem 1. For $z \in \mathcal{D}$ we consider $x(\cdot, z, \varepsilon):\left[0, t_{(z, \varepsilon)}\right) \rightarrow \mathbb{R}^{n}$ the solution of (1) such that $x(0, z, \varepsilon)=z$. From hypothesis $(H), t_{\left(z_{\alpha}, 0\right)}>T$ for every $\alpha \in V$. So as a consequence of Theorem 8.3 of [1] there exists a neighborhood of $U$ of $\mathcal{Z}$ and $0<\varepsilon_{2}<\varepsilon_{1}$ such that, for all $(z, \varepsilon) \in U \times\left(-\varepsilon_{2}, \varepsilon_{2}\right), t_{(z, \varepsilon)}>T$. Hence we can consider the function $f: U \times\left(-\varepsilon_{2}, \varepsilon_{2}\right) \rightarrow \mathbb{R}^{n}$, given by

$$
f(z, \varepsilon)=x(T, z, \varepsilon)-z .
$$

It is easy to see that system (1) for $\varepsilon=\bar{\varepsilon} \in\left(-\varepsilon_{2}, \varepsilon_{2}\right)$ has a periodic solution passing through $\bar{z} \in U$ if and only if $f(\bar{z}, \bar{\varepsilon})=0$.

To study the zeros of (17) is equivalent to study the zeros of the $\mathcal{C}^{m+1}$ function

$$
g(z, \varepsilon)=Y(T, z)^{-1} f(z, \varepsilon)
$$

On the other hand, we have that

$$
x(t, z, \varepsilon)=x(t, z, 0)+\varepsilon y_{1}(t, z)+\varepsilon^{2} \frac{y_{2}(t, z)}{2}+\varepsilon^{3} \frac{y_{3}(t, z)}{6}+\mathcal{O}\left(\varepsilon^{4}\right),
$$


IMPROVING THE AVERAGING THEORY FOR COMPUTING PERIODIC SOLUTIONS 11

where $y_{1}, y_{2}$ and $y_{3}$ are given in (8). For more details on these expression (19) see Lemma 1 of $[15,16]$. Thus

(20)

$$
\begin{aligned}
g(z, \varepsilon) & =Y(T, z)^{-1}(x(t, z, 0)-z)+\varepsilon Y(T, z)^{-1} y_{1}(T, z)+\varepsilon^{2} Y(T, z)^{-1} \frac{y_{2}(T, z)}{2} \\
& +\varepsilon^{3} Y(T, z)^{-1} \frac{y_{3}(t, z)}{6}+\mathcal{O}\left(\varepsilon^{4}\right) \\
& =g_{0}(z)+\varepsilon g_{1}(z)+\varepsilon^{2} g_{2}(z)+\varepsilon^{3} g_{3}(z)+\mathcal{O}\left(\varepsilon^{4}\right) .
\end{aligned}
$$

The same observation made about the value of $m$ for the expression (16) is applied for the expressions (19) and (20).

In order to apply Lemma 3 to function (18) we compute

$$
g_{0}\left(z_{\alpha}\right)=Y_{\alpha}(T)^{-1}\left(x\left(T, z_{\alpha}, 0\right)-z_{\alpha}\right)=0,
$$

and

$$
\begin{aligned}
\frac{\partial g_{0}}{\partial z}\left(z_{\alpha}\right)= & Y_{\alpha}(T)^{-1}\left(\frac{\partial x}{\partial z}\left(T, z_{\alpha}, 0\right)-I d\right) \\
& =Y_{\alpha}(T)^{-1}\left(Y_{\alpha}(T) Y_{\alpha}(0)^{-1}-I d\right) \\
& =Y_{\alpha}(0)^{-1}-Y_{\alpha}(T)^{-1}
\end{aligned}
$$

because $(\partial x / \partial z)(t, z, 0)$ is solution of $(3)$ such that $(\partial x / \partial z)(0, z, 0)=I d$, which implies that $(\partial x / \partial z)(t, z, 0)=Y(t, z) Y(0, z)^{-1}$.

From here the proof follows applying Lemma 3.

Proof of Proposition 2. First of all we define the following functions,

$$
\begin{aligned}
A_{0}(t)= & \int_{0}^{t} e^{s+\sin s} d s, A_{1}(t)=\int_{0}^{t} e^{-s-\sin s} A_{0}(s) d s, A_{2}(t)=\int_{0}^{t} A_{0}(s) \sin s d s, \\
A_{3}(t)= & \int_{0}^{t} A_{0}(s)^{2} \sin s d s, A_{4}(t)=\int_{0}^{t} A_{0}(s) \cos s \sin s d s, \\
A_{5}(t)= & \int_{0}^{t} A_{0}(s) A_{2}(s) \sin s d s, A_{6}(t)=\int_{0}^{t} A_{0}(s) A_{3}(s) \sin s d s, \\
A_{7}(t)= & \int_{0}^{t} A_{2}(s) \sin s d s, A_{8}(t)=\int_{0}^{t} A_{3}(s) \sin s d s, \\
A_{9}(t)= & \int_{0}^{t} A_{0}(s) A_{0}(-s) \sin s d s, A_{10}(t)=\int_{0}^{t} e^{-s-\sin s} A_{0}(s) \sin ^{2} s d s, \\
A_{11}(t)= & \int_{0}^{t} A_{1}(s) \sin ^{2} s d s, A_{12}(t)=\int_{0}^{t} e^{-s-\sin s} \sin ^{2} s d s .
\end{aligned}
$$


Now we shall describe the different elements which appear in the statement of Theorem 1 in the particular case of the differential system (9). Firstly, $T=2 \pi$, $\mathcal{D}=\mathbb{R}^{3}, x=(u, v, w), F_{0}(t, x)=(w, w,(1+\cos t) w), F_{1}(t, x)=\left(\mu v^{2} \sin t, \eta u^{2}\right.$ $\sin t, 0), F_{2}(t, x)=(\nu, \nu, \bar{\nu})$, and $F_{3}(t, x)=(0,0, \xi \sin t)$. The solution of the unperturbed system starting in $z=\left(u_{0}, v_{0}, w_{0}\right)$ is given by

$$
x(t, z, 0)=\left(u_{0}+w_{0} A_{0}(t), v_{0}+w_{0} A_{0}(t), w_{0} e^{t+\sin t}\right) .
$$

Thus for $w_{0}=0, x\left(t, u_{0}, v_{0}, 0,0\right)=\left(u_{0}, v_{0}, 0\right)$ is a $T$-periodic solution. So $\alpha=\left(u_{0}, v_{0}\right), \beta(\alpha) \equiv 0, k=2$ and $n=3$. Now we take the parameters $r_{1}>0$ and $r_{2}>0$ arbitrarily small and arbitrarily large, respectively. Let $V$ be the open and bounded subset of the plane $w_{0}=0$ given by

$$
V=\left\{\left(u_{0}, v_{0}, 0\right) \in \mathbb{R}^{3}: r_{1}<\sqrt{u_{0}^{2}+v_{0}^{2}}<r_{2}\right\} .
$$

Therefore, in our case the set

$$
\mathcal{Z}=\left\{z_{\alpha}=(\alpha, \beta(\alpha)), \alpha \in \bar{V}\right\}=\left\{\left(u_{0}, v_{0}, 0\right) \in \mathbb{R}^{3}: r_{1} \leq \sqrt{u_{0}^{2}+v_{0}^{2}} \leq r_{2}\right\} .
$$

Taking $Y_{\alpha}(t)=\frac{\partial x}{\partial z}\left(t, z_{\alpha}, 0\right)$ we obtain that

$$
Y_{\alpha}(0)^{-1}-Y_{\alpha}(2 \pi)^{-1}=\left(\begin{array}{ccc}
0 & 0 & e^{-2 \pi} A_{0}(2 \pi) \\
0 & 0 & e^{-2 \pi} A_{0}(2 \pi) \\
0 & 0 & 1-e^{2 \pi}
\end{array}\right)
$$

So

$$
\Gamma_{\alpha}=\left(\begin{array}{c}
e^{-2 \pi} A_{0}(2 \pi) \\
e^{-2 \pi} A_{0}(2 \pi)
\end{array}\right) \quad \text { and } \quad \Delta_{\alpha}=1-e^{2 \pi}
$$

We note that $\Gamma_{\alpha}$ and $\Delta_{\alpha}$ do not depend on $\alpha$, and it is easy to check that they are not null matrices.

Using the formulae (7) and (8) we compute

$$
g_{0}(u, v, w)=\left(e^{-2 \pi} w A_{0}(2 \pi), e^{-2 \pi} w A_{0}(2 \pi), w\left(1-e^{-2 \pi}\right)\right)
$$

and

$$
\begin{aligned}
y_{1}(t, z)= & \left(\mu\left(2 v w A_{2}(t)+w^{2} A_{3}(t)+v^{2}(1-\cos t)\right), \eta\left(2 u w A_{2}(t)\right.\right. \\
& \left.\left.+w^{2} A_{3}(t)+u^{2}(1-\cos t)\right), 0\right)
\end{aligned}
$$

So

$$
g_{1}(z)=\left(\mu w\left(2 v A_{2}(2 \pi)+w A_{3}(2 \pi)\right), \eta w\left(2 u A_{2}(2 \pi)+w A_{3}(2 \pi)\right), 0\right) .
$$


Using the formulae (5) and (6) we obtain that $\gamma_{1}(u, v)=0$ and $f_{1}(u, v)=(0,0)$. In this case the expressions of $\gamma_{2}$ and $f_{1}$ become simpler.

$$
f_{2}(\alpha)=\frac{1}{2} \Gamma_{\alpha} \gamma_{2}(\alpha)+\pi g_{2}\left(z_{\alpha}\right) \quad \text { and } \quad \gamma_{2}(\alpha)=-2 \Delta_{\alpha}^{-1} \pi^{\perp} g_{2}\left(z_{\alpha}\right)
$$

Again from (8) we compute $y_{2}(t, z)=\left(y_{2}^{1}(t, z), y_{2}^{2}(t, z), y_{2}^{3}(t, z)\right)$ where

$$
\begin{aligned}
y_{2}^{1}(t, z)= & \nu t-\bar{\nu}\left(A_{0}(t) A_{0}(-t)+A_{1}(t)\right)+4 \mu \nu u^{2} v \sin ^{4} \frac{t}{2}+2 \mu \eta w\left(u ^ { 2 } \left(A_{2}(t)\right.\right. \\
& \left.\left.-A_{4}(t)\right)+2 u w A_{5}(t)+w^{2} A_{6}(t)+2 u v A_{7}(t)+t v w A_{8}(t)\right), \\
y_{2}^{1}(t, z)= & \nu t-\bar{\nu}\left(A_{0}(t) A_{0}(-t)+A_{1}(t)\right)+4 \mu \nu u v^{2} \sin ^{4} \frac{t}{2}+2 \mu \eta w\left(v ^ { 2 } \left(A_{2}(t)\right.\right. \\
& \left.\left.-A_{4}(t)\right)+2 v w A_{5}(t)+w^{2} A_{6}(t)+2 u v A_{7}(t)+t u w A_{8}(t)\right), \\
y_{2}^{3}(t, z)= & -\bar{\nu} e^{t+\sin t} A_{0}(-t) .
\end{aligned}
$$

So from (7)

$$
\begin{aligned}
g_{2}(z)= & \left(\nu \pi-\frac{\bar{\nu}}{2} A_{1}(2 \pi)+\mu \eta w\left(u^{2}\left(A_{2}(2 \pi)-A_{4}(2 \pi)\right)+2 u w A_{5}(2 \pi)\right.\right. \\
& \left.+w^{2} A_{6}(2 \pi)+2 u v A_{7}(2 \pi)+2 \pi v w A_{8}(2 \pi)\right), \nu \pi-\frac{\bar{\nu}}{2} A_{1}(2 \pi) \\
& +\mu \eta w\left(v^{2}\left(A_{2}(2 \pi)-A_{4}(2 \pi)\right)+2 v w A_{5}(2 \pi)+w^{2} A_{6}(2 \pi)\right. \\
& \left.\left.+2 u v A_{7}(2 \pi)+2 \pi u w A_{8}(2 \pi)\right),-\frac{\bar{\nu}}{2} A_{0}(-2 \pi)\right) .
\end{aligned}
$$

Using now the formulae (21) we obtain

$$
\gamma_{2}(u, v)=\frac{\bar{\nu} A_{0}(-2 \pi)}{1-e^{-2 \pi}}
$$

and

$$
\begin{aligned}
f_{2}(u, v)= & \left(\pi \nu+\frac{\bar{\nu} A_{0}(2 \pi) A_{0}(-2 \pi)}{2\left(e^{2 \pi}-1\right)}-\frac{\bar{\nu} A 1(2 \pi)}{2},\right. \\
& \left.\pi \nu+\frac{\bar{\nu} A_{0}(2 \pi) A_{0}(-2 \pi)}{2\left(e^{2 \pi}-1\right)}-\frac{\bar{\nu} A 1(2 \pi)}{2}\right)=(0,0) .
\end{aligned}
$$

The last equality was obtained by substituting the value of $\bar{\nu}$ given in (10). 
Now using the formulae (8) and (6) we compute, respectively,

$$
\begin{aligned}
g_{3}(u, v, 0)= & \left(-\xi A_{10}(2 \pi)-\mu v\left(2 \pi \nu+\bar{\nu}\left(A_{9}(2 \pi)+A_{11}(2 \pi)\right),-\xi A_{10}(2 \pi)\right.\right. \\
& -\mu u\left(2 \pi \nu+\bar{\nu}\left(A_{9}(2 \pi)+A_{11}(2 \pi)\right), \xi A_{12}(2 \pi)\right), \\
\gamma_{3}(u, v)= & \frac{6 \xi A_{12}(2 \pi)}{e^{-2 \pi}-1}
\end{aligned}
$$

So from (5) we obtain

$$
f_{3}(u, v)=M\left(\begin{array}{l}
u \\
v
\end{array}\right)-\xi \chi
$$

where

$$
M=\left(\begin{array}{cc}
0 & 2 \pi \mu \nu K \\
2 \pi \eta \nu K & 0
\end{array}\right), \chi=\left(\begin{array}{c}
A_{10}(2 \pi)+\frac{A_{0}(2 \pi) A_{12}(2 \pi)}{e^{2 \pi}-1} \\
A_{10}(2 \pi)+\frac{A_{0}(2 \pi) A_{12}(2 \pi)}{e^{2 \pi}-1}
\end{array}\right) \approx\left(\begin{array}{l}
4.178 \\
4.178
\end{array}\right)
$$

and

$$
\begin{aligned}
K & =\frac{\left(e^{2 \pi}-1\right)\left(A_{1}(2 \pi)+A_{9}(2 \pi)+A_{11}(2 \pi)\right)-\left(A_{0}(2 \pi)+e^{2 \pi} A_{2}(2 \pi)\right) A_{0}(-2 \pi)}{A_{0}(2 \pi) A_{0}(2 \pi)+\left(e^{2 \pi}-1\right) A_{1}(2 \pi)} \\
& \approx 0.225 .
\end{aligned}
$$

From the hypotheses we have that $\operatorname{det} M \neq 0$. Hence there exists a solution $\left(u^{*}, v^{*}\right)$ of the linear system $f_{3}(u, v)=0$ such that $\operatorname{det}\left(D f_{3}\left(u^{*}, v^{*}\right)\right) \neq 0$. The proof follows by computing $\left(u^{*}, v^{*}\right)=M^{-1} \chi$ and applying Theorem (1).

\section{ACKNOWLEDGEMENTS}

We thank to the referees for their comments and suggestions which help us to improve the presentation of this paper.

The first author is partially supported by a MINECO/FEDER grant MTM200803437 and MTM2013-40998-P, an AGAUR grant number 2013SGR-568, an ICREA Academia, the grants FP7-PEOPLE-2012-IRSES 318999 and 316338, FEDERUNAB-10-4E-378. The second author is partially supported by a FAPESPBRAZIL grant 2013/16492-0. The two authors are also supported by a CAPES CSF-PVE grant 88881.030454/ 2013-01.

\section{REFERENCES}

[1] H. Amann, Ordinary differential equations. An introduction to nonlinear analysis, de Gruyter Studies in Mathematics 13, Walter de Gruyter \& Co., Berlin, 1990. 
[2] A. Buica And J. Llibre, Averaging methods for finding periodic orbits via Brouwer degree, Bulletin des Sciences Mathemàtiques 128 (2004), 7-22.

[3] A. Buiç̆, J.P. FrançOISE And J. Llibre, Periodic solutions of nonlinear periodic differential systems with a small parameter, Communication on Pure and Applied Analysis 6 (2007), 103-111.

[4] A. Buiç̆, J. Giné AND J. LliBRE, A second order analysis of periodic solutions for nonlinear periodic differential systems with a small parameter, Physica D 241 (2012), 528533.

[5] A. Buică, J. Giné And J. LliBre, Periodic solutions for nonlinear differential systems: The second order bifurcation function, Topol. Methods Nonlinear Anal. 43 (2014), 403-419.

[6] C. Chicone, Lyapunov-Schmidt reduction and Melnikov integrals for bifurcation of periodic solutions in coupled oscillators, J. Differential Equations 112 (1994), 407-447.

[7] M.B.H. Rhouma And C. Chicone, On the continuation of periodic orbits, Methods Appl. Anal. 7 (2000), 85-104.

[8] R.D. EuzÉBio AND J. Llibre, Periodic solutions of El Niño model through the Vallis differential systems, Discrete and Continuous Dynamical Systems, Serie A 35 (2014), 34553469 .

[9] J. Giné, M. Grau And J. Llibre, Averaging theory at any order for computing periodic orbits, Physica D 250 (2013), 58-65.

[10] J.L.G. Guirao, J. Llibre And J.A. VerA, Generalized van der Waals Hamiltonian: periodic orbits and $C^{1}$ noninterability, Physical Review E 85 (2012), 036603.

[11] J.L.G. Guirao, J. Llibre And J.A. VerA, Periodic orbits of Hamiltonian systems: applications to perturbed Kepler problems, Chaos, Solitons and Fractals 57 (2013), 105111.

[12] L. JimÉnez And J. Llibre, Periodic orbits and non integrability of Henon-Heiles systems, J. of Physics A: Math. Theor. 44 (2011), 205103, pp. 14.

[13] J. Llibre, A.C. Mereu And M.A. Teixeira, Limit cycles of the generalized polynomial Liénard differential equations, Math. Proceed. Camb. Phyl. Soc. 148 (2009), 363-383

[14] J. Llibre, D.D. Novaes And M.A. Teixeira, On the periodic solutions of a perturbed double pendulum, São Paulo J. Math. Sci. 5 (2011), no. 2, 317-330.

[15] J. Llibre, D.D. Novaes And M.A. Teixeira, Higher order averaging theorem for finding periodic solutions via Brouwer degree, Nonlinearity 27 (2014), 563-583.

[16] J. Llibre, D.D. Novaes AND M.A. TeiXeirA, Corrigendum: Higher order averaging theory for finding periodic solutions via Brouwer degree, Nonlinearity 27 (2014), 2417.

[17] J. Llibre, S. Rebollo-Perdomo And J. Torregrosa, Limit cycles bifurcating from isochronous surfaces of revolution in $\mathbb{R}^{3}$, J. Math. Anal. and Appl. 381 (2011), 414-426.

[18] J. Llibre, And G. Swirszcz, On the limit cycles of polynomial vector fields, Dyn. Cont. Discrete Impuls. Syst. 18 (2011), 203-212.

[19] J. Llibre, AND X. Zhang, Hopf bifurcation in higher dimensional differential systems via the averaging method, Pacific J. of Math 240 (2009), 321-341

[20] J. Llibre, And X. Zhang, On the Hopf-zero bifurcation of the Michelson system, Nonlinear Analysis, Real World Applications 12 (2011), 1650-1653

[21] I.G. Malkin, Some problems of the theory of nonlinear oscillations, (Russian) Gosudarstv. Izdat. Tehn.-Teor. Lit., Moscow, 1956.

[22] M. Roseau, Vibrations non linéaires et théorie de la stabilité, (French) Springer Tracts in Natural Philosophy, Vol.8 Springer-Verlag, Berlin-New York, 1966.

[23] F. SPIRIG Bifurcation equation for planar systems of differential equations, (German summary) Z. Angew. Math. Phys. 39 (1988), no. 4, 504-517. 
[24] J. A. Sanders F. Verhulst and J. Murdock, Averaging Methods in Nonlinear Dynamical Systems, Second edition, Applied Mathematical Sciences 59, Springer, New York, 2007.

[25] M. SpIvak, Calculus On Manifolds: A Modern Approach To Classical Theorems Of Advanced Calculus, Addison-Wesley Publishing Company, 1965.

[26] F. Verhulst, Nonlinear Differential Equations and Dynamical Systems, Universitext, Springer, 1991.

1 Departament de Matematiques, Universitat Autònoma de Barcelona, 08193 Bellaterra, Barcelona, Catalonia, Spain

E-mail address: jllibre@mat.uab.cat, ddnovaes@mat.cat.uab

2 Departamento de Matemática, Universidade Estadual de Campinas, Rua Sérgio Buarque de Holanda, 651, Cidade Universitária Zeferino Vaz, 13083-859, CampINAS, SP, BRAZIL

E-mail address: dnovaes@ime.unicamp.com 